Dieses Dokument ist eine Zweitveröffentlichung (Verlagsversion) / This is a self-archiving document (published version):

Markus Hundt, Alexander Lasch

Das Niederdeutsche im Rahmen einer Sprachgeschichte des Deutschen Erstveröffentlichung in / First published in:

Jahrbuch für Germanistische Sprachgeschichte. 2015, 6 (1), S. 4 - 17. De Gruyter. ISSN: 18697046.

DOI: https://doi.org/10.1515/jbgsg-2015-0004

Diese Version ist verfügbar / This version is available on:

https://nbn-resolving.org/urn:nbn:de:bsz:14-qucosa2-748627 


\section{Das Niederdeutsche im Rahmen einer Sprachgeschichte des Deutschen}

\section{Hinführung}

Das Nd. spielt in der institutionalisierten Sprachgeschichtsforschung des Deutschen seit über 150 Jahren als Gegenstand und als Varietät, die bis ins östliche Baltikum als Verkehrssprache galt, eine marginale Rolle (vgl. dazu Sanders 1982; Debus 1996; Wirrer 1998; Stellmacher 2000 und 2004). Die Auseinandersetzung mit den Varietäten des Nordens hat zum Ziel, das Bewusstsein dafür zu entwickeln, dass man mit der Verkehrssprache zusammen bisher die historische Entwicklung eines ganzen Sprach- und Kulturraums vernachlässigte (vgl. Michelsen 1993; Debus 1996 und Stellmacher 2004). Auf der 5. Jahrestagung der Gesellschaft für Germanistische Sprachgeschichte wurden die verstreuten Studien zu diesem Bereich gebündelt. Die Fachtagung mit dem Titel „Deutsch im Norden. Varietäten des norddeutschen Raumes im Spiegel der germanistischen Sprachgeschichtsschreibung“, die vom 25. bis 27. September 2014 an der Christian-AlbrechtsUniversität zu Kiel durchgeführt wurde, bot für diejenigen, die sich in diesem Forschungsgebiet betätigen, ein anregendes Diskussionsforum. Blickt man auf die Forschungslandschaft zum Nd., so kann man feststellen, dass sich nur vergleichsweise wenige Arbeiten mit der Sprachgeschichte des Nd. befassen (vgl. Sanders 1982; Stellmacher 2000). Eher ist davon zu sprechen, dass „Aspekte“ einer Sprachgeschichte vorgelegt worden sind (vgl. Eickmanns 2003; Gessinger 2003; Mattheier 2003; Peters 2003a und b; Rösler 2003; Scheuermann 2003). Noch immer ist die Frage von Stellmacher (1998) aktuell, ob, und wenn ja warum, „für die Darstellung der niederdeutschen Sprache eigene Prinzipien“ gelten.

Die Varietäten des nrddt. Raumes (vom And. bis zu den heutigen nrddt. Varietäten der Alltagssprache) nehmen in der sprachhistorischen Forschung eine nachgeordnete Rolle ein, ein ganzer Sprach- und Kulturraum - nämlich der des Ond. (Stellmacher 1980 und Rösler 2003) - liegt im blinden Fleck der Forschung. Davon betroffen sind schließlich auch Konzeptionen eines balt. Kulturraums, obwohl diese ebenfalls schon seit 20 Jahren vorgetragen werden (u.a. Michelsen 1993 und Debus 1996). Diese Orientierung auf den Sprach- und Kulturraum ist vermutlich weniger in der historischen und kulturellen Entwicklung des gesamten Sprachraumes sowie der Sprachentwicklung selbst begründet, sondern wohl auch darin, dass sich die Eliten Norddeutschlands nach dem Untergang 
der Hanse im 16. Jh. an Prestigevarietäten des Südens orientierten. Nicht zuletzt dürfte die Orientierung der Literaturgeschichts- und Grammatikschreibung an hd. Varietäten seit dem 17. Jh. wesentlich dazu beigetragen haben, dass die Varietäten des nrddt. Raumes eher selten im Fokus der Forschung stehen (vgl. dazu kritisch Peters 1998 und Stellmacher 1998).

An zwei kurzen Fallbeispielen soll im Folgenden diskutiert werden, wie das Nd. als Gegenstand durch die am Hd. orientierte Grammatikschreibung seit dem 18. Jh. bewertet wird, um die Notwendigkeit eines Perspektivenwechsels zu plausibilisieren, und darüber hinaus die internationale Forschung zum Sprachund Kulturraum des Baltikums anzustoßen und zu fördern. Die 5. Jahrestagung der Gesellschaft für Germanistische Sprachgeschichte („Deutsch im Norden. Varietäten des norddeutschen Raumes im Spiegel der germanistischen Sprachgeschichtsschreibung“) stellt mit den in diesem Band versammelten Beiträgen einen Schritt in die Richtung des angesprochenen Perspektivwechsels dar.

\section{Sprachgeschichte des Klanges: Von der Ursprünglichkeit des Niederdeutschen}

Im Philologischen Tresespiel (Mattheson 1752 / 1975) rechnet Johann Mattheson (1681-1764) wahrscheinlich ungehört und sehr spät mit Johann Christoph Gottsched (1700-1766) ab (vgl. im Detail Lasch 2010). Der Streit, in dem Mattheson mit dem Tresespiel seine Position noch einmal deutlich herausstellt, nimmt seinen Anfang in der Auseinandersetzung mit Johann Adolph Scheibe (1708-1776) über die - ohne das freilich so zu benennen - rechte,Fachsprache der Musik‘. Scheibe veröffentlichte die Zeitschrift Der Critische Musicus (1737-1740) in direkter Konkurrenz zur Critica Musica (1722-1725) Matthesons und bat seinen Lehrer Gottsched um eine Rezension in dessen Beyträgen zur critischen Historie der Deutschen Sprache. Diese erschien 1739. Darin lobt Gottsched, dass der Musicus in der „reinen und unvermengten Muttersprache abgehandelt sei“ und das hebe ihn von anderen ,musikalischen Büchern“ ab, die „wegen der besonderen Schreibart, in welche sie meistentheils, wo nicht alle eingehüllet sind, dunkel, wo nicht gar unverständlich“ seien (Beyträge St. 23, Nr. VIII, 453-465, hier 465). Mattheson, der ungefragt als Gewährsmann genannt wurde, besteht auf einer Entgegnung, die zwei Jahre später in den Beyträgen erschien. Allerdings wird der Beitrag Matthesons durch Gottsched in 13 Anmerkungen kommentiert, die durch eine sehr kleinliche und insgesamt befremdliche obermeisterliche Art auffallen. Das Tresepiel antwortet - elf Jahre später - auf diese Demütigung. Aus dieser Widerrede sei hier nur auf einen Aspekt abgehoben, nämlich die von Gottsched anvisierte 
,Norm einer deutschen Standardsprache‘, so würde man heute sagen, die er als normgebende Instanz zu etablieren sucht (die aber freilich mit einer Sprache der Musik oder Fachsprache der Musik als funktionalen Varietäten nichts gemein hat). Dieser Frage widmet Mattheson ein ganzes Kapitel:

\begin{abstract}
Sagen sie [die Obersachsen] so [beedes für beydes]? Ey! das ist recht artig! Wir bemerken demnach, im Vorbeygehen, die Wahrheit dessen [...], daß 1) ein anders sey, eine Sprache zu schreiben; ein andres aber, dieselbe zu reden; wobey das Letztere vorgeht. Auch siehet man 2) deutlich hieraus, wie sehr die niedersächsische, zärtere Ursprache der obersächsischen in den Nacken stößt. (Mattheson 1752 / 1975, 46)
\end{abstract}

Mattheson, der „,bedeutendste Kritiker, Ästhetiker, Polemiker, ja Enzyklopädist der deutschen M[usik]g[eschichte] des 18. Jahrhunderts“ (Turnow 2001, 1812), tritt nicht nur für das phonographische Prinzip im Bereich der Orthographie ein, sondern widersetzt sich, polemisierend, dem Prinzip eines nationalen Standards auf der Basis des Hd., indem er das Nsächs. als die „zärtere Ursprache“ stilisiert. Die Basis für diese Beurteilung bildet der Klang als entscheidende Kategorie: Das Hd., so würde man seine Argumentation heute nachzeichnen, sei durch die zweite Lautverschiebung im Bereich des Konsonantismus, verunreinigt‘ worden:

Glaubwürdig ist es, daß die ungeschliffene Gothen dieses leidige ch in der Bulgarey ausgeheckt haben: denn diejenige hohe Mundart, die so voll davon ist, erkennet ja öffentlich, für ihren reinen Ursprung die vermischten, trüben Quellen der Gothen, Allemannen und Franken. Das kann man gern zugeben; auch zur Lust wol ein wenig beleuchten. (Mattheson 1752 / 1975, Fol. ${ }^{\star \star} 3^{\mathrm{v}}$ und ${ }^{\star \star} 4^{\mathrm{r}}$ )

Außerdem habe es einen Hang im Vokalismus zu Diphthongen - das Nd. hingegen behält im Konsonantismus die germ. (,reinen') Formen bei und gönnt sich im Bereich des Vokalismus keine Eskapaden:

Dann nun jeder Vogel am liebsten und besten so singet, wie ihm der Schnabel gewachsen ist: einer hoch, scharf und rauch, der ander tief, gelinde und glatt, ${ }^{*}$ so hat bis diese Stunde der Uhu fast grösser Recht haben wollen, als die Nachtigall [...]. (Mattheson 1752 / 1975, Fol. * ${ }^{5}$ )

In der Fußnote $\left(^{\star}\right)$ zu glatt wird erläutert: „Ich sage wohlbedächtlich, mit lachendem Munde, glatt, an Statt: platt. Dieses letztere ist wohl [sc. in den üblichen Darstellungen!] ein Druckfehler allenthalben; wie zu glauben“. Sollte man, so Mattheson, daher nicht für das Nd. als „zärtere Ursprache“ das Recht reklamieren, Basis für eine deutsche Kultursprache zu sein?

Der Streit zwischen Gottsched und Mattheson dokumentiert zum einen im Ausschnitt den Diskurs über die Etablierung einer ,Norm des Standarddeutschen“ im 18. Jh., zum anderen auch die Stabilisierung von kommunikativen Subdomänen, die sich dem Zugriff der (selbst ernannten) Norminstanzen entziehen und 
verweigern, wie z.B. die der ,neuen Fachsprachen', für die Mattheson hier exemplarisch steht (vgl. zur Diskussion um den Normbegriff auch Hundt 2009). Eine Beschäftigung mit der Architektur dieser Diskurse ist lohnend und in der sprachhistorischen Forschung noch weitestgehend Neuland. Das letzte Urteil, das über das Tresespiel Matthesons gefällt wurde, stammt von Siegfried Kross:

„Was [im Tresespiel] über den Vorzug des Niederdeutschen vor dem schon durch Luthers Bibelübersetzung zur Grundlage des Schriftdeutschen gewordenen Obersächsischen gesagt wird [...] oder zur Rückführung sowohl des Griechischen als des Lateinischen auf das Altsächsische vorgetragen wird, ist so unhaltbar und überholt, war es wohl auch schon zu seiner Zeit, daß man darüber kein Wort zu verlieren braucht.“ (Kross 1983, 342)

Kross mag fachlich recht haben. Dass es Mattheson aber überhaupt nicht auf einen sprachhistorisch motivierten Disput anlegte, sondern auf eine Polemik gegen die selbst ernannte Norminstanz (die Kross ohne Widerspruch zu akzeptieren scheint), wird nicht gesehen: Die Option darauf, dass man wie Mattheson ,das‘ Nd. als Varietät im Norden wertschätzen könnte, kommt überhaupt nicht in Betracht angesichts des ,Hochwerts einer überregionalen Standardsprache‘. Hier sollte man in der Forschung heute eher programmatisch von kulturellen Differenzen und Spannungen sprechen, die man nicht der Einfachheit halber, zumal in der Sprachgeschichtsschreibung nicht, dem Ideal der ,Norm eines nationalen sprachlichen Standards‘ opfert.

\section{Sal vnsrer Löve Vernöttinge syn: Zur Bewertung des Okkasionalismus Verknotigung}

Das Ännchen von Tharau ${ }^{1}$ hält für den Leser eine Textpassage bereit, die lexikalisch markiert ist: Krankheit, Verfolgung, Betrübnis und Pein / Soll unsrer Liebe Verknotigung sein. Als Sprachwissenschaftler hat man schnell verschiedene Hypothesen zur Hand, wie das auffällige Lexem Verknotigung zu erklären ist, wie z.B. diese: Man kann Verknotigung (noch) leidlich verstehen; vermutlich ist es ein Archaismus: Von der Wortbildung her betrachtet (Derivation: deverbales Substantiv mit -ung zum Verb verknot[ig]en) kann das Wort kaum sehr viel älter als 400 Jahre sein. Spricht man von einem Archaismus, muss das Wort irgendwann einmal üblich gewesen sein, über die Varietät und die kommunikative Reichweite

1 Zur Liedgeschichte und der Angabe der hier zitierten Belege vgl. vor allem Fischer 2007 mit weiterführenden Quellen und Editionen des Liedes, weiter Müller-Blattau 1945. 
kann man kaum Aussagen machen. Etwas eigenwillig und auffällig allerdings ist der artifizielle Ausbau des Stammes zu verknotigen statt *verknoten. Ist dafür das Versmaß einer vorliegenden Melodie ursächlich? Der Text, der uns mit dem nhd. Ännchen von Tharau (1778) vorliegt, stammt von Johann Gottfried Herder (1744-1803) (vgl. Haym 1880, Jäger 1969). Er übertrug die nd. Version des Liedes Anke von Tharaw (1636) (dazu besonders Andraschke 2002, 186-203), die Simon Dach (1605-1659) (vgl. Fischl 1909; Oesterley 1876; Flemming 1957) zugeschrieben wird. Die (mutmaßlich) erste Vertonung von 1642, die auch Herder gekannt haben dürfte, stammt von Heinrich Albert (1604-1651) (vgl. Dommer 1875; Dadelsen 1953 und Müller-Blattau 1954, 26-32, bes. 31f.). Der heute bekannte Satz ist wesentlich jünger (1827, Friedrich Silcher [1789-1860]; vgl. Weber 1892; Brusniak 2010). Belassen wir es also der Einfachheit halber zunächst dabei, dass bereits Herder bei der ,Übersetzung‘ aus dem Npr. ins Hd. einem Versmaß gefolgt ist und deshalb für die Übertragung Verknotigung statt *Verknotung wählte. Herder übertrug folgende Verse:

Kranckheit, Verfälgung, Bedröfnös on Pihn,

Sal vnsrer Löve Vernöttinge syn.

Folgte Herder dem Text Dachs in der Übertragung wortgenau, dann hätte er hier *Vernietunge statt Verknotigung, also „Vernietung“ statt „Verknotung“, gesetzt es gehört aber zu den Freiheiten des Umgangs mit Texten, dass diese variiert, angepasst und geändert werden können. Auch ist das Bild der „Vernietung“ in unserem Kulturkreis eher unüblich, die „Verknotung“ von Liebenden oder besser in der Institution der Ehe Verbundenen dagegen eine gebräuchliche Figur. Von einem Archaismus wird man folglich nicht sprechen können, sondern von einem Okkasionalismus. Dennoch ist die Frage nicht, warum Herder das Bild von der „Vernietung“ ersetzte, sondern welche Hinweise es auf Motive gibt, weshalb Dach es in der npr. Fassung verwendete.

Simon Dach (1605-1659) wurde in Wittenberg und Magdeburg ausgebildet und studierte später in Königsberg. Ab 1639 war er Professor für Dichtkunst an der dortigen Universität, obwohl er erst 1640 promoviert wurde - seine guten Beziehungen zu Georg Wilhelm von Brandenburg (1595-1640) mögen dafür genau so ausschlaggebend gewesen sein wie seine Begeisterung für Literatur: Er gilt als Mittelpunkt der Kürbishütte, einer Sprachgesellschaft, wie sie zur Zeit des 30jährigen Krieges Konjunktur im Reich hatten (vgl. speziell zum opr. Dichterkreis Kelletat 1986, bes. 383-386). Sprachgesellschaften, wie der Palmenorden (auch Fruchtbringende Gesellschaft), setzten sich zum Ziel, das Deutsche als Sprache für Kunst und Literatur zu befördern, auszubauen und zu pflegen - allerdings im Gegensatz zu heutigen sprachpflegerischen Bemühungen (die eher sprachkonservatorisch orientiert sind) in einem progressiven Sinne. Mit dem Ausweis des 
Alters der deutschen Sprache, der Vielgestaltigkeit und des Variantenreichtums verfolgte man das Interesse, das Deutsche neben den rom. Sprachen und dem noch immer dominierenden Lat. in Wissenschaft und Kunst zu etablieren. Dabei schoss das ein oder andere Mitglied mit seinen Sprachspielen in Lehnübersetzung, Synonymfindung und grammatischen Spekulationen in einzelnen Fällen zwar über das Ziel hinaus (genannt werden hierfür gern die nicht akzeptierten Lehnübersetzungen Philipp von Zesens), dennoch darf die Auseinandersetzung mit der deutschen Sprache im 17. Jh. als Vorläufer der modernen Sprachwissenschaft gelten und hatte nicht $\mathrm{zu}$ verachtende Einflüsse auf die Entwicklung der deutschen Literatur - als „Spracharbeit“ bezeichnet man diese Praxis (vgl. Hundt 2000, zur Wissenskonstitution durch Spracharbeit auch Hundt 2011). Simon Dach ist diesem Kreis intellektueller ,Spracharbeiter` zuzurechnen; sein Talent lag vor allem im massenhaften Anfertigen von für das 17. Jh. typischer Gebrauchslyrik, die zu jedem Anlass angemessen, zur gesellschaftlichen Repräsentation absolut notwendig war und daher tausendfach überliefert (und bisher kaum hinreichend untersucht) ist. Zu ihren Kennzeichen zählt auf der einen Seite das ,Entlangdichten' an textuellen Mustern, die der klassischen Rhetorik entlehnt sind, auf der anderen Seite eine Liebe für das Sprachspiel, zu dem auch das Setzen unkonventioneller Metaphern und Synonyme gehört. „Vernietunge“ ist möglicherweise ein Sprachspiel dieser Art. Es ist davon auszugehen, dass Herder dieses Sprachspiel erkannte. Obwohl er das unübliche Bild der „Vernietunge“ durch das der „Verknotigung“ ersetzte, trägt er mit der okkasionellen Bildung mit der artifiziellen zusätzlichen Nebensilbe in „Verknotigung“ der Vorlage Rechnung - er behält deren barocke sprachlich-verspielte Markiertheit bei.

An diesem Sprachspiel - oder besser an Herder - kommen schließlich auch die Bearbeiter des Deutschen Wörterbuchs offensichtlich nicht vorbei, sie gestehen „Verknotigung“ neben „Verknotung“ ein eigenes Lemma zu:

verknotigung, f. enge, feste verknüpfung mittels eines knotens:

käm alles wetter gleich auf uns zu schlahn,

wir sind gesinnt bei einander zu stahn.

krankheit, verfolgung, betrübnisz und pein

soll unsrer liebe verknotigung sein.

HERDER z. lit. u. kunst 8, 483 (1821),

ein vermuthlich von HERDER erfundenes wort mit anlehnung an das Dachische

sal unsrer löwe vernöttinge syn.

DACH 420 Österley,

welches genau übersetzt unserem vernietung entspräche.

(DWB, digitale Ausgabe, Art. Verknotigung)

Dabei werden sowohl Ursprung als auch Urheber der Neuschöpfung benannt, zugleich die wortwörtliche Alternative angegeben - ob im adverbialen „genau“ 
eine Wertung transportiert wird, soll hier nicht weiter erörtert werden; die Möglichkeit, dass Herder hier ein Sprachspiel fortgesetzt haben könnte (und nicht nur sklavisch dem Versmaß folgte, wofür es auch andere Alternativen gegeben hätte), scheint indes nicht auf. Interessant im Kontext des weiter oben angesprochenen Perspektivwechsels in der Sprachgeschichtsforschung ist, dass die von Dach ganz offenkundig betriebene Spracharbeit im Nd. (Npr.) von der Forschung bislang nicht zur Kenntnis genommen worden ist, wohingegen eine okkasionelle Nachbildung eines nd. Sprachspiels als ,Übersetzung“ ins Hd. mehr oder weniger sofort Eingang ins Deutsche Wörterbuch gefunden hat. Ein weiterer interessanter Aspekt in diesem Zusammenhang ist das Pronomen „unser“. Damit werden zwei mögliche Referenzen eröffnet: eine temporale (,in unserem aktuellen Gebrauch: Vernietung') und eine sprachtypologische (,in unserem Hd.: Vernietung`). Möglich sind bei den Grimms (und den späteren Bearbeitern des Wörterbuchs im 20. Jh.) nur auf den ersten Blick beide Lesarten. Die erste Lesart scheidet schon deshalb aus, weil das Deutsche Wörterbuch ohnehin auch etymologische Interessen verfolgte und ein Verweis auf ältere Sprachstufen und andere Varietäten in der Regel auch direkt benannt wird. Die zweite Lesart hingegen ist die plausiblere. Sie würde durch das notwendige „Übersetzen“ gestützt. Doch für diesen thematischen Aufriss zur Einleitung ins Thema würden Diskussionen um den Stellenwert der Varietäten des Deutschen bei den Sprach(geschichts) forschern des 19. (und 20.) Jhs. zu weit führen, ganz abgesehen von der Frage, ob das Nd. als Varietät dem Deutschen überhaupt zuzurechnen sei oder nicht: Genau diese Diskussionen und Fragen standen neben anderen im Mittelpunkt der Tagung „Deutsch im Norden“. ${ }^{2}$

2 Das Lemma im Deutschen Wörterbuch macht aber das gänzlich anders gelagerte lexikografische Interesse im Gegensatz zu heutigen lexikologischen und lexikografischen Standards noch viel deutlicher. Ein ,hochsprachlicher' Beleg reichte aus, um Verknotigung mit einem eigenen Lemma zu würdigen - im heutigen Duden suchte man vergeblich nach einem solchen Eintrag. Heute ist der frequente Gebrauch eines Wortes ausschlaggebend dafür, ob es in ein Wörterbuch aufgenommen wird oder nicht - die Frequenz oder Auftretenshäufigkeit lässt sich aber erst seit 15 bis 20 Jahren dank neuerer technischer Entwicklungen zuverlässig für große Teile des Wortschatzes ermitteln. So kann man sich auf der einen Seite freuen, dass Verknotigung als okkasionelle Bildung im Deutschen im Lied Ännchen von Tharau und im Deutschen Wörterbuch die Zeit überdauert. Auf der anderen Seite kann man dankbar dafür sein, dass sich heute Wörterbucharbeit nicht mehr auf literarische Hochsprache als alleinige Quelle stützt, sondern den je gegenwärtigen Sprachgebrauch zu erfassen beabsichtigt - , unsere Sprache‘, die wir, ganz wertneutral, hier und jetzt verwenden. Zum dritten wird an diesem Beispiel deutlich, wie stark sich die Auffassung von dem, was Deutsch sei, im Wandel befindet. Zum vierten kann man gelassen auf Einflüsse auf das Deutsche blicken und die Frage, ob man bewusst die Sprachentwicklung 
Wie an den beiden knapp diskutierten illustrativen Fallbeispielen deutlich geworden sein sollte, gibt es komplette Gegenstandsbereiche, die durch negative Bewertung und gesteuerten Wissenstransfer überhaupt nie ins Bewusstsein der Fachdiskussion rücken und damit als Gegenstand etabliert werden konnten. Das ist umso bedauerlicher, als gerade über das Nd. als einer Gruppe von Varietäten Anschluss gesucht werden kann an die Forschungen zum Sprach- und Kulturraum des Baltikums.

\section{Aufbau des Bandes}

Die Tagung „Deutsch im Norden. Varietäten des norddeutschen Raumes im Spiegel der germanistischen Sprachgeschichtsschreibung“ warf verschiedene Perspektiven auf das eingangs skizzierte Thema.

Der Band zur Tagung ist in insgesamt drei Themenbereiche gegliedert. Die Beiträge der ersten Sektion „Exemplarische Analysen“ setzen sich mit der sprachhistorischen Entwicklung und dem Kontakt zwischen Hd. und Varietäten des nrddt. Raumes (in der Forschung vgl. dazu u.a. Peters 1998 und 1999) auseinander. Dabei werden Quellen aus den verschiedensten Textsortenbereichen berücksichtigt, die bisher selten Gegenstand der Forschung waren - so bspw. Quellen des Ond., welches sich in den Gebieten östlich der Elbe als Ausgleichsvarietät nach dem 11. Jh. etabliert und in der Forschung vollkommen unterrepräsentiert ist (für einige wenige Beispiele siehe u.a. Gernentz 1991 oder Föllner 1999). Immer wieder wird dabei auch die ,alte‘ Frage nach dem Status der Varietäten des nrddt. Raumes aufgegriffen und an Quellenmaterial diskutiert: Wurde das Nd. durch das Hd. (als Schriftvarietät) verdrängt oder wurde das Hd. durch die Sprachbenutzer des Nd. favorisiert? Welchen Status haben vor diesem Hintergrund die Varietäten im nrddt. Raum vom 18. Jh. bis zur Gegenwart? (vgl. dazu bisher u.a. Mellado 1998; Menke 1998 und Stellmacher 2004).

In der zweiten Sektion „Diskussion methodischer Zugänge“ diskutieren verschiedene Beiträge methodisch neue Zugänge zur Erforschung der Varietäten des nrddt. Raumes im Hinblick auf ihre Entwicklung (vgl. in der Forschung etwa Appel 2006 und 2012 oder auch die neuerliche Konzentration auf die [historische] Syntax des Nd. u.a. bei Langhanke 2012 oder Berg 2013).

Beiträge in der Sektion „Das Niederdeutsche in der Grammatikographie“ bilden die Klammer zu den einleitenden kurz diskutierten Fallbeispielen und

(negativ) prägen könne: Selbst mit Herder und Stützung durch die Grimms hat es Verknotigung nicht geschafft, sich im Sprachgebrauch zu etablieren. 
runden mit Fragen nach der (Be-)Wertung der Varietäten des nrddt. Raumes in der Grammatikschreibung und Sprachwissenschaft den Band ab (vgl. in der Forschung zu diesem Themenkreis weiter z.B. Wirrer 1992 und 1998; Kremer 1997; Goossens 2000 und Arendt 2010).

Den Beiträgen der Sektionen geht als weiterer Teil der Einleitung der einführende Überblick von RoBERT PETERS „Zur Sprachgeschichte des norddeutschen Raumes“ voraus.

Die „Exemplarischen Analysen“ - soweit das möglich ist folgen sie einer chronologischen Ordnung - eröffnet der Beitrag von KATRIN DRÄGER mit dem namenskundlichen Beitrag „Witte versus Schwarz. Zur Verhochdeutschung niederdeutscher Familiennamen“. Sie zeigt, dass die Verhochdeutschung deutscher Familiennamen eine deutliche Abstufung von Südosten nach Nordwesten aufweist und vor allem deappellativische Familiennamen betrifft, wenn sie eine hd. Entsprechung aufweisen. Im Anschluss daran illustriert THомAs GLONING anhand des mnd. Gaerde der suntheit (1492) Vertextungspraxen frühneuzeitlicher Kräuterbücher und weist dezidiert auf die gemeinsamen etablierten und stabilen Muster der Textorganisationspraxen in den Bereichen früher Fachsprachlichkeit hin. THOMAs BIEBERSTEDT legt in einer differenzierten variablenlinguistischen Analyse dar, wie sich im städtischen Kanzleischriftum (fokussiert auf Wismar und Stralsund) zentrale Formen des Mnd. (Phonographematik, Morphologie, Lexik) in vier Zeiträumen im 14. und 15. Jh. verändert haben. Beide Städte werden in ihrem Schreibusus in Beziehung gesetzt sowohl zu sechs weiteren Städten des Ostseeraums als auch zur lübischen Schreibsprache, die bei beiden Orten eine Orientierung für die jeweilige Variantenwahl ist. Peter Hinkelmanns befasst sich mit der nd. Schriftlichkeit in Form von Inschriften auf Grabplatten aus Greifswald aus der Zeit vor 1700. Zwar dominierten bis 1520 hier lat. Inschriften, mit dem Aufkommen der Reformation erlebte jedoch das Nd. als Inschriftensprache einen Aufschwung. Ab der zweiten Hälfte des 16. Jhs. ist dann eine nd.-hd. Mehrsprachigkeit in den Inschriften zu konstatieren, die dann mit der Durchsetzung des Hd. in den Inschriften nach 1650 als abgeschlossen betrachtet werden kann. LuISE CzAJKowsKi untersucht den Sprachwechsel vom Nd. zum Hd. am Beispiel des Stadtbuchs von Alsleben aus dem 15. Jh. Sie kann mit korpuslinguistischen Analyseverfahren überzeugend darlegen, wie sich in diesem Beispielfall die Schreibsprache vom Nd. zum Hd. im Zeitraum von 1454 bis 1490 verändert hat. MARc Schutzeichel und Renata Szczepaniak gehen anhand von Hexenverhörprotokollen aus dem 16. und 17. Jh. der Frage nach, ob und wie ggf. der Sprachwechsel vom Nd. zum Hd. auch mit der Durchsetzung der Großschreibung in einen Zusammenhang gebracht werden kann. Sie stellen interessante Ergebnisse in Bezug auf die Durchsetzungsrichtung der satzinternen Großschreibung im Unterschied zum bekannten Drei-Phasen-Modell von Gabrielsson vor. Im Bei- 
trag von BIRGIT CHRISTEnsEN wird die komplexe Gemengelage des Übergangs vom Nd. zum Hd. in verschiedenen kirchlichen Textsorten im 17. Jh. am Beispiel der Städte Sønderborg und Tønder nachgezeichnet. Es zeigt sich, dass auch hier ein allmählicher, z.T. durch bestimmte Faktoren verzögerter Übergang vom Nd. zum Hd. festzustellen ist, der vor allem dadurch motiviert ist, dass dem Hd. ein höheres Sozialprestige zugewiesen wird, was Christensen bis zum Gebrauch einzelner Varianten (nd. dochter vs. hd. Tochter) ad nominem nachweisen kann. Die erste Sektion beschließt der Beitrag von Viola Wilcken. Sie beschäftigt sich mit der Frage, ob und wie Missingsch aus überlieferten literarischen Texten in historischer Perspektive rekonstruiert werden kann. Anhand eines Korpus von 43 Texten aus einem Zeitraum von 1805 bis zur Gegenwart kann sie eine ganze Reihe von linguistischen Variablen eruieren, die sowohl typisch für das Missingsch sind als auch die Veränderungen im Missingschen aufzeigen; so werden nicht nur die bekannten stabilen Merkmale, sondern auch sukzessive im Abbau befindliche und neue, d.h. erst nach 1930 greifbare Merkmale dargestellt.

Die zweite Sektion „Diskussion neuer methodischer Zugänge“ eröffnet der Beitrag von INGRID SCHRÖDER. Sie fragt nach theoretischen und methodischen Möglichkeiten, spezifische mündliche nrddt. Sprachverhältnisse hinsichtlich ihrer räumlichen, sozialen und funktionalen Vielfalt modellieren zu können. Sie wendet sich dazu im Beispiel der sprachlichen Situtation in Hamburg, Altona und dem näheren Umland zwischen 1600 und 1800 zu. Der Beitrag von NiNA BARTSch und Simone Schultz-BAlluff ist in der historischen Syntax angesiedelt. Anhand von Parallelüberlieferungen eines Textes können die Autorinnen sowohl in der fokussierten Varietät des Mnd. als auch in weiteren Varietäten syntaktische Spezifika und syntaktische Entwicklungen im Bereich der Nebensatzeinleitung herausarbeiten. Dadurch werden schließlich auch die besonderen Überlieferungszusammenhänge im nd. Sprachraum sichtbar und plausibilisiert. Einen weiteren Beitrag zur historischen Syntax des Mnd.bieten KATHARINA DreEsSEn und SARAH IHDEn. Sie greifen auf das Referenzkorpus Mittelniederdeutsch / Niederrheinisch (1200-1650) zurück und analysieren drei Phänomene der Syntax: die Wortstellung in Haupt- und Nebensätzen, den Verbalkomplex insgesamt und die Verbalklammer. Es zeigt sich, dass bei diesen Phänomenen insbesondere die jeweilige Textsorte syntaxrelevant ist. MATTHIAs ScHuLz wendet sich in seinem Beitrag einem Quellenfundort zu, der bislang in der germanistischen Sprachgeschichte noch wenig Beachtung gefunden hat, den städtischen (Kirchen-) Bibliotheken. Er erläutert am Beispiel von Kirchenbibliotheken aus dem 15. / 16. Jh. (fokussiert auf die Bestände der Kirchenbibliothek in Barth), wie die darin enthaltenen Buchbestände zusammengesetzt waren und welche Aufschlüsse man aus dieser Quellengruppe für den Sprachwechsel vom Nd. zum Hd. in der Frühen Neuzeit gewinnen kann. Abschließend zeigt der Beitrag von LARS 
VORBERGER, wie sich der Sprachwechselprozess vom Nd. zum Hd. im Brandenb. in Wechselwirkung mit dem Berl. vollzogen hat. Dazu greift er auf das entsprechende aussagekräfte Material der Wenkerbögen aus 17 brandenb. und 8 heutigen Berliner Ortspunkten zurück.

Die beiden Beiträge der dritten Sektion „Das Niederdeutsche in der Grammatikographie“ zeigen an zwei Fallbeispielen auf, dass die Frage nach dem Stellenwert des Nd. in der hd. dominierten Sprachgeschichte ein Desiderat ist. RoBERT LANGHANKE zeichnet in seinem Beitrag die Bemühungene Klaus Groths um die Etablierung einer nnd. Schriftlichkeit nach und geht dabei auch auf die sprachreflexiven Ansätze Groths ein, die sich z.B. in der Entwicklung einer nd.-spezifischen Orthographie zeigten. KARSTEN RINAS, dessen Beitrag diese Sektion und damit auch den Band beschließt, richtet den Blick auf die Grammatikographie des ausgehenden 17. Jhs. Er umreißt anhand von verschiedenen Bearbeitungen der Grammatik von Bödiker (Grundsätze der Teutschen Sprache), welche z.T. modern anmutende Vorstellungen Bödiker in Bezug auf die Stellung von Dialekten und hier insbes. dem Nd. hatte. Im Gegensatz $\mathrm{zu}$ anderen Zeitgenossen (Pudor) verteidigt Bödiker - so das Fazit Rinas` - das Nd.

\section{Zusammenfassung}

Insgesamt zeigen die hier vorgelegten Beiträge, dass sich der eingangs angesprochene Perspektivenwechsel hin zu einer Sprachgeschichte der deutschen Varietäten lohnt: Ein typologisches Kriterium, nämlich dass das Nd. anders als das Hd. die zweite Lautverschiebung nicht mitgemacht habe, darf heute nicht mehr dazu führen, einen ganzen Varietätenraum aus der sprachhistorischen Forschung auszuklammern. Die Beiträge in diesem Band zeigen in Detailstudien und im Blick auf das Ganze, wie plausibel sich Sprachwandel gerade dann nachvollziehen lässt, wenn die Chance genutzt wird, alle Varietäten des Deutschen in die Überlegungen einzubeziehen. Es ist auch im Hinblick auf den balt. Sprach- und Kulturraum heute nicht mehr damit getan, die Hauptentwicklungen des Sprachwechsels vom Nd. zum Hd. in der Frühen Neuzeit zu benennen. Die Analysen von Einzelentwicklungen einerseits und sprachsystembezogenen (z.B. Syntax) und raumbezogenen (z.B. Ond.) Besonderheiten andererseits stellen auch wegen der teils schwierigen Quellenlage neue methodische Ansätze zur Diskussion und bringen Ergebnisse ans Licht, die die Sprachgeschichte des Deutschen wesentlich bereichern. 


\section{Literatur}

Andraschke, Peter (2002), „Der volkstümliche Herder: Ausblick in die Moderne. Eine musikalische Dokumentation“, in: Ders. / Helmut Loos (Hrsg.), Ideen und Ideale. Johann Gottfried Herder in Ost und West, Freiburg im Breisgau, 167-207.

Appel, Heinz-Wilfried (2006), Untersuchungen zur Syntax niederdeutscher Dialekte:

Forschungsüberblick, Methodik und Ergebnisse einer Korpusanalyse (Literatur - Sprache - Region, 7), Frankfurt am Main u.a.

Appel, Heinz-Wilfried (2012), Kleines Valenzwörterbuch niederdeutscher Verben, Frankfurt am Main u.a.

Arendt, Birte (2010), Niederdeutschdiskurse: Spracheinstellungen im Kontext von Laien, Printmedien und Politik (PSQ, 224), Berlin.

Berg, Kristian (2013), Morphosyntax nominaler Einheiten im Niederdeutschen (Germanistische Bibliothek, 49), Heidelberg.

Brusniak, Friedhelm (2010), „Silcher, Friedrich“, in: Neue Deutsche Biographie, 24, 412-414, online abrufbar unter http://www.deutsche-biographie.de/pnd118614339.html (letzter Aufruf 11.02.2014).

Dadelsen, Georg von (1953), „Albert, Heinrich“, in: Neue Deutsche Biographie, 1, 138-139, online abrufbar unter http://www.deutsche-biographie.de/pnd121418952.html (letzter Aufruf 11.02.2014).

Debus, Friedhelm (1996), Von Dünkirchen bis Königsberg: Ansätze und Versuche zur Bildung einer niederdeutschen Einheitssprache, Göttingen.

Deutsches Wörterbuch von Jacob und Wilhelm Grimm, 16 Bde., Leipzig 1854-1961, online abrufbar unter: http://goo.gl/aQHtp (letzter Aufruf 01.01.2013).

Dobrovolskij, Dmitrij O. / Elisabeth Piirainen (1992), „Zum Weltmodell einer niederdeutschen Mundart im Spiegel der Phraseologie“, in: NdW, 32, 137-169.

Dommer, Arrey von (1875), „Albert, Heinrich“, in: Allgemeine Deutsche Biographie, online abrufbar unter http://www.deutsche-biographie.de/pnd121418952.html? anchor=adb (letzter Aufruf 11.02.2014).

Eickmans, Heinz (2003), „Aspekte einer niederrheinischen Sprachgeschichte“, in: Werner Besch u.a. (Hrsg.), Sprachgeschichte. Ein Handbuch zur Geschichte der deutschen Sprache und ihrer Erforschung, Teilbd. (HSK, 2, 3), 2., vollst. neu bearb. und erw. Aufl., Berlin / New York, 2699-2712.

Elmentaler, Michael (1998), „Diachrone Schreibsprachenforschung und historische Dialektologie des Niederdeutschen“, in: NdJb, 121, 25-58.

Fischer, Michael (2007), „Ännchen von Tharau“, in: Populäre und traditionelle Lieder. Historisch-kritisches Liederlexikon des Deutschen Volksliedarchivs, online abrufbar unter http://www.liederlexikon.de/lieder/aennchen_von_tharau (letzter Aufruf 11.02.2014).

Fischl, Oskar (1909), „Motive des Properz in Simon Dachs ,Anke von Tharaw““, in: Euph., 8, 11-16.

Flemming, Willi (1957), „Dach, Simon“, in: Neue Deutsche Biographie, 3, 464-465, online abrufbar unter http://www.deutsche-biographie.de/pnd11852321X.html (letzter Aufruf 11.02.2014).

Föllner, Ursula (Hrsg.) (1999), Zum Status des Niederdeutschen in Sachsen-Anhalt: Kontinuität und Wandel in Vergangenheit und Gegenwart (Schriftenreihe der Arbeitsstelle Niederdeutsch, 3), Oschersleben.

Gernentz, Hans-Joachim (1991), „Zur Verwendung des Niederdeutschen in Dramen des Rostocker Raums um 1600“, in: Beiträge zur Erforschung der deutschen Sprache, 10, 216-222. 
Gessinger, Joachim (2003), „Aspekte einer Sprachgeschichte des Brandenburgischen“, in: Werner Besch u.a. (Hrsg.), Sprachgeschichte. Ein Handbuch zur Geschichte der deutschen Sprache und ihrer Erforschung, Teilbd. (HSK, 2, 3), 2., vollst. neu bearb. und erw. Aufl., Berlin / New York, 2674-2699.

Goossens, Jan (2000), „Zur Lage des Niederdeutschen und ihrer Erforschung“, in: Heinz Eickmans / Loek Geeraedts / Robert Peters (Hrsg.), Ausgewählte Schriften zur niederländischen und deutschen Sprach- und Literaturwissenschaft, Münster u.a., 377-395.

Gottsched, Johann Christoph (Hrsg.) (1732-1744), Beyträge zur critischen Historie der Deutschen Sprache, Poesie und Beredsamkeit, zitiert nach dem reprografischen Nachdruck im Verlag Georg Olms, Hildesheim 1970.

Haym, Rudolf (1880), „Herder, Johann Gottfried“, in: Allgemeine Deutsche Biographie online abrufbar unter http://www.deutsche-biographie.de/pnd118549553.html?anchor $=$ adb (letzter Aufruf 11.02.2014).

Hermann-Winter, Renate (2013), Sprachatlas für Rügen und die vorpommersche Küste, Rostock. Hundt, Markus (2000), Spracharbeit im 17. Jahrhundert. Studien zu Georg Philipp Harsdörffer, Justus Georg Schottelius und Christian Gueintz (Studia linguistica Germanica, 57), Berlin / New York.

Hundt, Markus (2009), „Normverletzung und neue Normen“, In: Marek Konopka / Bruno Strecker (Hrsg.), Deutsche Grammatik - Regeln, Normen, Sprachgebrauch (JIdS 2008), Berlin / New York, 117-140.

Hundt, Markus (2011), „Diskursivierung von Wissen durch Sprache - der multimodale Ansatz von Georg Philipp Harsdörffer in den Frauenzimmer Gesprächspielen“, in: Thorsten Burkard u.a. (Hrsg.), Politik - Ethik - Poetik. Diskurse und Medien frühneuzeitlichen Wissens (Diskursivierung von Wissen in der Frühen Neuzeit, 1), Berlin, 177-200.

Jäger, Hans-Wolf (1969), „Herder, Johann Gottfried“, in: Neue Deutsche Biographie, 8, 595-603, online abrufbar unter http://www.deutsche-biographie.de/pnd118549 553.html (letzter Aufruf 11.02.2014).

Kelletat, Alfred (1986), Simon Dach und der Königsberger Dichterkreis, Stuttgart.

Kremer, Ludger (1997), „Das niederdeutsche Paradox: Ausbau und Verlust einer Regionalsprache“, in: Germanistische Mitteilungen, 45-46, 5-13.

Kross, Siegfried (1983), „Mattheson und Gottsched“, in: George J. Buelow / Hans Joachim Marx (Hrsg.), New Mattheson Studies, Cambridge u.a., 327-344.

Langhanke, Robert u.a. (Hrsg.) (2012), Niederdeutsche Syntax (GL, 220), Hildesheim / Zürich / New York.

Lasch, Alexander (2010), „Ein Hamburger verbittet sich das Oberdeutsche. Sprachhistorisches zur Auseinandersetzung zwischen Mattheson und Gottsched“", in: Wolfgang Hirschmann / Bernhard Jahn (Hrsg.), Johann Mattheson als Vermittler und Initiator. Wissenstransfer und die Etablierung neuer Diskurse in der ersten Hälfte des 18. Jahrhunderts, Hildesheim / Zürich / New York, 407-421.

Lauf, Raphaela (1996), „,Regional markiert': großräumliche Umgangssprache(n) im niederdeutschen Raum“, in: NdJb, 119, 193-218.

Lenz, Alexandra N. (Hrsg.) (2009), Low Saxon dialects across borders = Niedersächsische Dialekte über Grenzen hinweg (ZDL Beiheft, 139), Stuttgart.

Mattheier, Klaus J.( 2003), „Aspekte einer rheinischen Sprachgeschichte“, in: Werner Besch u.a. (Hrsg.), Sprachgeschichte. Ein Handbuch zur Geschichte der deutschen Sprache und ihrer Erforschung, Teilbd. (HSK, 2, 3), 2., vollst. neu bearb. und erw. Aufl., Berlin / New York, 2712-2729. 
Mattheson, Johann (1752), Philologisches Tresespiel, Reprint Leipzig 1975.

Mellado Blanco, Carmen (1998), „Zum Status des Niederdeutschen heute und gestern: Dialekt oder Sprache?“, in: WW, 48, 420-433.

Menke, Hubertus (1998), „Niederdeutsch: eigenständige Sprache oder Varietät einer Sprache?“, in: Eva Schmitsdorf / Nina Haiti / Barbara Meurer (Hrsg.), Lingua Germanica: Studien zur deutschen Philologie. Jochen Splett zum 60. Geburtstag, Münster u.a., 171-184.

Michelsen, Friedrich W. (1993), „Bilden die niederdeutschen Mundarten eine soziokulturelle Einheit? Anmerkungen zu einer aktuellen Frage“, in: NdW, 33, 125-137.

Müller-Blattau, Joseph (1945), „Wege des Volksliedes III. Ännchen von Tharau“, in: Die Musik, 26, 490-497.

Müller-Blattau, Joseph (1954), „Heinrich Albert und das weltliche Barocklied“, in: Günter Kraft (Hrsg.), Festschrift zur Ehrung von Heinrich Albert (1604-1651), Weimar: s.n.

Oesterley, Hermann (1876), „Dach, Simon“, in: Allgemeine Deutsche Biographie, online abrufbar unter http://www.deutsche-biographie.de/pnd11852321X.html?anchor=adb (letzter Aufruf 11.02.2014).

Peters, Robert (1998), „Zur Sprachgeschichte des niederdeutschen Raumes“, in: ZfdPh, 117, Sonderheft Regionale Sprachgeschichte, 108-127.

Peters, Robert (1999), „Zur Rolle des Niederdeutschen bei der Entstehung des Neuhochdeutschen“, in: Walter Hoffmann (Hrsg.), Das Frühneuhochdeutsche als sprachgeschichtliche Epoche. Werner Besch zum 70. Geburtstag, Frankfurt am Main u.a., 161-173.

Peters, Robert (2003a), „Aspekte einer Sprachgeschichte des Sassischen“, in: Werner Besch u.a. (Hrsg.), Sprachgeschichte. Ein Handbuch zur Geschichte der deutschen Sprache und ihrer Erforschung, Teilbd. (HSK, 2, 3), 2., vollst. neu bearb. und erw. Aufl, Berlin / New York, 2651-2662.

Peters, Robert (2003b), „Aspekte einer Sprachgeschichte des Westfälischen“, in: Werner Besch u.a. (Hrsg.), Sprachgeschichte. Ein Handbuch zur Geschichte der deutschen Sprache und ihrer Erforschung, Teilbd. (HSK, 2, 3), 2., vollst. neu bearb. und erw. Aufl., Berlin / New York, 2640-2650.

Polenz, Peter von (1991-1999), Deutsche Sprachgeschichte vom Spätmittelalter bis zur Gegenwart, 3 Bde, Berlin / New York.

Prowatke, Christa (1985), „Gesprächsbücher des 17. Jahrhunderts und ihre sprachwissenschaftliche Auswertung. Ein Beitrag zur Schreibung des Niederdeutschen“, in: Beiträge zur Erforschung der deutschen Sprache, 5, 66-79.

Prowatke, Christa (1998), Zur Ausbildung der Graphie in einer regionalen mittelniederdeutschen Literatursprache unter Berücksichtigung der schreibsprachlichen Situation im gesamten deutschen Sprachraum am Beispiel ausgewählter Quellen der Rostocker Druckerei des Ludwig Dietz (1512-1559), Rostock, Univ., Diss.

Rösler, Irmtraud (2003), „Aspekte einer Sprachgeschichte des Ostniederdeutschen“, in: Werner Besch u.a. (Hrsg.), Sprachgeschichte. Ein Handbuch zur Geschichte der deutschen Sprache und ihrer Erforschung, Teilbd. (HSK, 2, 3), 2., vollst. neu bearb. und erw. Aufl., Berlin / New York, 2699-2712.

Sanders, Willy (1982), Sachsensprache, Hansesprache, Plattdeutsch: sprachgeschichtliche Grundzüge des Niederdeutschen, Göttingen.

Scheuermann, Ulrich (2003), „Aspekte einer Sprachgeschichte des Ostfälischen“, in: Werner Besch u.a. (Hrsg.), Sprachgeschichte. Ein Handbuch zur Geschichte der deutschen Sprache 
und ihrer Erforschung, Teilbd. (HSK, 2, 3), 2., vollst. neu bearb. und erw. Aufl., Berlin / New York, 2663-2674.

Schröder, Ingrid (1999), „Niederdeutsche Zaubersprüche: Konstanz und Variation“, in: Peter Wagener (Hrsg.), Sprachformen. Deutsch und Niederdeutsch in europäischen Bezügen. Festschrift für Dieter Stellmacher zum 60. Geburtstag, Stuttgart, 81-92.

Skvajrs, Ekaterina R (2009), Die Hanse in Novgorod: Sprachkontakte des Niederdeutschen mit dem Russischen (NdSt, 53), Köln / Weimar / Wien.

Spiewok, Wolfgang (1989), „Die mittelalterliche Literaturlandschaft im niederdeutschen Sprachraum“, in: Wolfgang Spiewok / Danielle Buschinger (Hrsg.), Mittelalter-Studien II. Göppingen, 267-283.

Spiewok, Wolfgang (1998a), „Die mittelalterliche Literaturlandschaft im niederdeutschen Sprachraum“, in: Ders., Mittelalterliche Literatur up plattdütsch, Greifswald, 11-25.

Spiewok, Wolfgang (1998b), „Der tanzende Tod in niederdeutschen Städten“, in: Ders., Mittelalterliche Literatur up plattdütsch, Greifswald, 88-97.

Stellmacher, Dieter (1980), „Ostniederdeutsch“, in: Hans Peter Althaus / Helmut Henne / Herbert Ernst Wiegand (Hrsg.), Lexikon der Germanistischen Linguistik, Berlin / New York, 464-468.

Stellmacher, Dieter (1998), „Gelten für die Darstellung der niederdeutschen Sprachgeschichte eigene Prinzipien?“, in: $P B B, 120,368-375$.

Stellmacher, Dieter (2000), Niederdeutsche Sprache (GeLe, 26), 2., überarb. Aufl., Berlin.

Stellmacher, Dieter (Hrsg.) (2004), Sprachkontakte: niederländisch, deutsch und slawisch östlich von Elbe und Saale (Wittenberger Beiträge zur deutschen Sprache und Kultur, 3), Frankfurt am Main u.a.

Taubken, Hans (1990), „,Westniederdeutsch“ und ,Nordniedersächsisch‘: zur gegenwärtigen Verwirrung der Terminologie der Dialekteinteilung des Niederdeutschen“, in: Robert Damme u.a. (Hrsg.), Franco-Saxonica: münstersche Studien zur niederländischen und niederdeutschen Philologie. Jan Goossens zum 60. Geburtstag, Neumünster, 203-237.

Turnow, Hans (2001), „Art. Johann Mattheson“, in: Friedrich Blume (Hrsg.), Die Musik in Geschichte und Gegenwart. Allgemeine Enzyklopädie der Musik, elektronische Ausgabe der ersten Auflage (1949-1986) (Digitale Bibliothek, 69), Berlin, VIII, 1797-1816.

Wallmeier, Nadine (2013), Sprachliche Muster in der mittelniederdeutschen Rechtssprache. Zum Sachsenspiegel und zu Stadtrechtsaufzeichnungen des 13. bis 16. Jahrhunderts (NdSt, 55), Köln / Weimar / Wien.

Weber, o. N. (1892), „Silcher, Friedrich“, in: Allgemeine Deutsche Biographie, online abrufbar unter http://www.deutsche-biographie.de/pnd118614339.html?anchor=adb (letzter Aufruf 11.02.2014).

Wiesinger, Peter (1983), „Die Einteilung der deutschen Dialekte“, in: Werner Besch u.a. (Hrsg.), Dialektologie. Ein Handbuch zur deutschen und allgemeinen Dialektforschung, Teilbd. (HSK, 1, 2), Berlin / New York, 807-900.

Wirrer, Jan (1992), „,So herrli klingt mi keen Musik un singt keen Nachdigal': Niederdeutsch gestern, Niederdeutsch heute; Perzeptionen und Bewertungen“, in: NdW, 32, 109-135.

Wirrer, Jan (1998), „Zum Status des Niederdeutschen“, in: ZGL, 26, 308-340.

Wollin, Lars (1990), „Niederdeutsch in Skandinavien: 3. Sigunta August 1989“, in: ZfdPh, 109, 420-427. 